\title{
MP CBM-Z V1.0: design for a new Carbon Bond Mechanism Z (CBM-Z) gas-phase chemical mechanism architecture for next-generation processors
}

\author{
Hui Wang ${ }^{1}$, Junmin Lin $^{2, a}$, Qizhong Wu ${ }^{1}$, Huansheng Chen ${ }^{3}$, Xiao Tang ${ }^{3}$, Zifa Wang ${ }^{3}$, Xueshun Chen ${ }^{3}$, \\ Huaqiong Cheng ${ }^{1}$, and Lanning Wang ${ }^{1}$ \\ ${ }^{1}$ College of Global Change and Earth System Science, Joint Center for Global Changes Studies, \\ Beijing Normal University, Beijing 100875, China \\ ${ }^{2}$ Intel (China) Corporation, Beijing 100013, China \\ ${ }^{3}$ State Key Laboratory of Atmospheric Boundary Layer Physics and Atmospheric Chemistry, \\ Institute of Atmospheric Physics, Chinese Academy of Sciences, Beijing 100029, China \\ ${ }^{a}$ now at: Artificial Intelligence Research Department, JD Corp., Beijing 100101, China
}

Correspondence: Qizhong Wu (wqizhong@bnu.edu.cn) and Huansheng Chen (chenhuansheng@mail.iap.ac.cn)

Received: 15 February 2018 - Discussion started: 24 April 2018

Revised: 13 November 2018 - Accepted: 22 January 2019 - Published: 20 February 2019

\begin{abstract}
Precise and rapid air quality simulations and forecasting are limited by the computational performance of the air quality model used, and the gas-phase chemistry module is the most time-consuming function in the air quality model. In this study, we designed a new framework for the widely used the Carbon Bond Mechanism Z (CBM-Z) gas-phase chemical kinetics kernel to adapt the single-instruction, multiple-data (SIMD) technology in next-generation processors to improve its calculation performance. The optimization implements the fine-grain level parallelization of CBM$\mathrm{Z}$ by improving its vectorization ability. Through constructing loops and integrating the main branches, e.g., diverse chemistry sub-schemes, multiple spatial points in the model can be operated simultaneously on vector processing units (VPUs). Two generation CPUs - Intel Xeon E5-2680 V4 CPU and Intel Xeon Gold 6132 - and Intel Xeon Phi 7250 Knights Landing (KNL) are used as the benchmark processors. The validation of the CBM-Z module outputs indicates that the relative bias reaches a maximum of $0.025 \%$ after $10 \mathrm{~h}$ integration with $-f p$-model fast $=1$ compile flag. The results of the module test show that the Multiple-Points CBM-Z (MP CBM-Z) resulted in 5.16× and $8.97 \times$ speedup on a single core of Intel Xeon E5-2680 V4 and Intel Xeon Gold 6132 CPUs, respectively, and KNL had a speedup of $3.69 \times$ compared with the performance of CBM-Z on the Intel Xeon E5-2680 V4 platform. For the single-node tests,
\end{abstract}

the speedup on the two generation CPUs can reach $104.63 \times$ and $198.50 \times$ using message passing interface (MPI) and $101.02 \times$ and $194.60 \times$ using OpenMP, and the speedup on the KNL node can reach $175.23 \times$ using MPI and $167.45 \times$ using OpenMP. The speedup of the optimized CBM-Z is approximately $40 \%$ higher on a one-socket KNL platform than on a two-socket Broadwell platform and about $13 \%$ $16 \%$ lower than on a two-socket Skylake platform. We also tested a three-dimensional chemistry transport model (CTM) named Nested Air Quality Prediction Model System (NAQPMS) equipped with the MP CBM-Z. The tests illustrate an obvious improvement on the performance for the CTM after adopting the MP CBM-Z. The results show that the MP CBM-Z leads to a speedup of 3.32 and 1.96 for the gas-phase chemistry module and the CTM on the Intel Xeon E5-2680 platform. Moreover, on the new Intel Xeon Gold 6132 platform, the MP CBM-Z gains $4.90 \times$ and $2.22 \times$ speedups for the gas-phase chemistry module and the whole CTM. For the KNL, the MP CBM-Z enables a $3.52 \times$ speedup for the gas-phase chemistry module, but the whole model lost $24.10 \%$ performance compared to the CPU platform due to the poor performance of other modules. In addition, since this optimization seeks to improve the utilization of the VPU, the model is more suitable for the new generation processors adopting the more advanced SIMD technology. The results of our tests already show that the benefit 
of updating CPU improved by about $47 \%$ by using the MP CBM-Z since the optimized code has better adaptability for the new hardware. This work improves the performance of the CBM-Z chemical kinetics kernel as well as the calculation efficiency of the air quality model, which can directly improve the practical value of the air quality model in scientific simulations and routine forecasting.

\section{Introduction}

Air pollution and its impacts on human health have attracted widespread attention all over the world, especially in developing countries (Gurjar et al., 2016; Zhang et al., 2017). As a useful tool for air quality problems, chemistry transport models (CTMs), are widely used in studies of air quality (Gao et al., 2016; Chen et al., 2015; Wu et al., 2014) and in establishing air quality forecasting (AQF) systems. As the core of the AQF system, a CTM requires a large number of computational resources to simulate the complex chemical and physical processes. To satisfy the demand of routine air quality forecasting in a timely manner, coarse spatial resolution and relatively simple processes are adopted in CTMs to minimize the use of computational resources. Meanwhile, other simulation studies with more complex processes are also limited by computational resources. Therefore, air quality studies can benefit significantly by improving the performance of the CTM used.

In a CTM, the most time-consuming module is the gasphase chemistry module (Wang et al., 2017). The gas-phase chemistry module is described as a system of ordinary differential equations (ODEs) to simulate the chemical kinetics of trace gases in an atmosphere model (Seinfeld and Pandis, 2012). Linford et al. (2009) reported that the Regional Acid Deposition Model version 2 (RADM2) (Zimmermann and Poppe, 1994; Chang et al., 1987), a chemical kinetics kernel, accounted for $90 \%$ of the computational time in the Weather Forecasting and Research/Chemistry (WRFchem) model (Grell et al., 2005). Another widely used chemical kinetics kernel, the Carbon Bond Mechanism version Z (CBM-Z) (Zaveri and Peters, 1999), accounts for approximately $68 \%$ of the computation time in the Global Nested Air Quality Prediction Model System (GNAQPMS) (Chen et al., 2015; Wang et al., 2017). Therefore, accelerating the gas-phase chemistry module can directly improve the performance of the CTM as well as the whole AQF system. The AQF system can also benefit from the performance improvement by adopting a higher model resolution and improving the frequency of air quality forecasting.

The performance of models improves with updated hardware. However, by reaching the bottleneck of power density and the thermal limitation of the silicon technology for a single-core design, frequent updating has not been an efficient way to improve the scientific model's perfor- mance. Additionally, multicore architecture and a heterogeneous computing architecture such as a Many Integrated Core (MIC) and a graphic processing unit (GPU) have become the hardware trend for high-performance computing (Xu et al., 2015; Lawrence et al., 2018). Meanwhile, to take full advantage of the advanced features of new processor architecture, the applications or the models must be redesigned or rewritten. Xu et al. (2015) rewrote the Princeton Ocean Model (POM) using Compute Unified Device ArchitectureC (CUDA-C) to port it from a CPU to a GPU platform. Linford et al. (2009) also tried to solve the computation bottleneck of RADM2 mentioned above by using a heterogeneous platform such as GPU-CPU. In addition, our previous work showed the primary optimizations we performed to accelerate the GNAQPMS on the new generation CPU and Intel MIC platforms (Knights Landing, KNL; Sodani et al., 2016) and had a significant performance improvement on both platforms, a $2.77 \times$ speedup on CPU and a $3.51 \times$ speedup on the KNL node (Wang et al., 2017). In this study, we redesign the code structure of the chemical kinetics kernel $\mathrm{CBM}-\mathrm{Z}$ to improve its vectorization performance on the CPU and KNL platforms, which significantly improves its performance by fully utilizing the single-instruction, multiple-data (SIMD) technology. We tested the performance of this optimized CBM-Z module as well as a regional CTM equipped with it. The code test only contained this single module, making it easier to let the CTM developers reuse the code.

Section 2.1 in this paper introduces the CBM-Z scheme, and Sect. 2.2 describes the new architecture we designed for CBM-Z. Since multiple spatial points were operated simultaneously in the optimized CBM-Z scheme, the optimized CBM-Z scheme was called the Multiple-Points CBM-Z Version 1.0 (MP CBM-Z V1.0). In Sect. 3.1, we present our benchmark platforms. In Sect. 3.2 and 3.3, we introduce the test cases and present the test results of single-model tests and CTM tests separately. The conclusions and discussions are given in Sect. 4.

\section{Method description}

CBM-Z is a core module in CTMs that simulates the complex gas-phase chemical processes in the atmosphere. In this module, too many options and poor load balancing within the model grid boxes make it a challenge to improve its performance on a vectorization level. This leads to poor performance of $\mathrm{CBM}-\mathrm{Z}$ on the new generation processors that are highly dependent on powerful vector processing units (VPUs). In our previous work, we conducted several optimizations on CBM-Z to enhance its vectorization and parallel performance (Wang et al., 2017). In this work, we attempt to further enhance its vector calculation ability by constructing a new structure, which makes the CBM-Z module suitable to be vectorized. The CBM-Z module was extracted as 
an individual box model to test its performance and improve code reusability.

\subsection{Description of CBM-Z}

CBM-Z is a lumped-structure photochemical mechanism that was developed to meet the needs of city-scale to globalscale tropospheric chemical simulations (Zaveri and Peters, 1999). The original scheme contains 67 species and 132 reactions. CBM-Z has been widely used in CTMs, e.g., the WRFChem (San José et al., 2015), the Nested Air Quality Prediction Model System (NAQPMS) (Wang et al., 2001) and the GNAQPMS. In the NAQPMS and GNAQPMSs, CBM$\mathrm{Z}$ was further modified by $\mathrm{Li}$ et al. (2012). It was updated to 76 species, and 28 heterogeneous reactions were added. The CBM-Z solver uses the modified backward Euler (MBE) solver developed by Feng et al. (2015), a faster and more robust algorithm which overcomes inflexibility and preserves the non-negativity.

The main control flow of CBM-Z is shown in Fig. 1. The IntegrateChemistry function is treated as the core function of the module. CBM-Z contains five chemistry sub-schemes. They are the Common Chemistry Scheme (COM), the Urban Chemistry Scheme (URB), the Biogenic Chemistry Scheme (BIO), the Marine Chemistry Scheme (MAR), and the Heterogeneous Chemistry Scheme (HET). The integration of different sub-schemes is used to satisfy the simulation of diverse scenarios and scales. The combination of sub-schemes relies on the concentration and emission of each chemical species in the specific model grid, which is implemented in the SelectGasRegime function. The variable iregime stores the return-value of SelectGasRegime and controls the subsequent calculation processes of CBM-Z. The possible values and the sub-schemes represented are shown in Table 1. The combinations include the COM and HET schemes, while other schemes are added when the concentration or emission of a corresponding species in a certain scheme are greater than zero. Compared with the algorithm computing all chemical interactions, this algorithm is helpful in saving computational resources on a simple core, while such irregular and unbalanced calculations lack well-structured loops and impede the vectorization of code. Besides the chemistry sub-schemes mentioned above, CBM-Z uses other functional branches, e.g., nocturnal and diurnal chemistry, and they impede the vectorization of the computation. The CBM$\mathrm{Z}$ also contains multiple unconstructed scalar operations. We partially integrated the scalar operations by using indirect indexing to construct loops for vectorization (Wang et al., 2017). However, this method required significant effort, and it only reconstructed a limited number of scalar operations. The CBM-Z module still contains many scalar operations. With multi-level control flow divergences and many scalar calculations, it is not feasible to perform automatic vectorization with an Intel compiler.

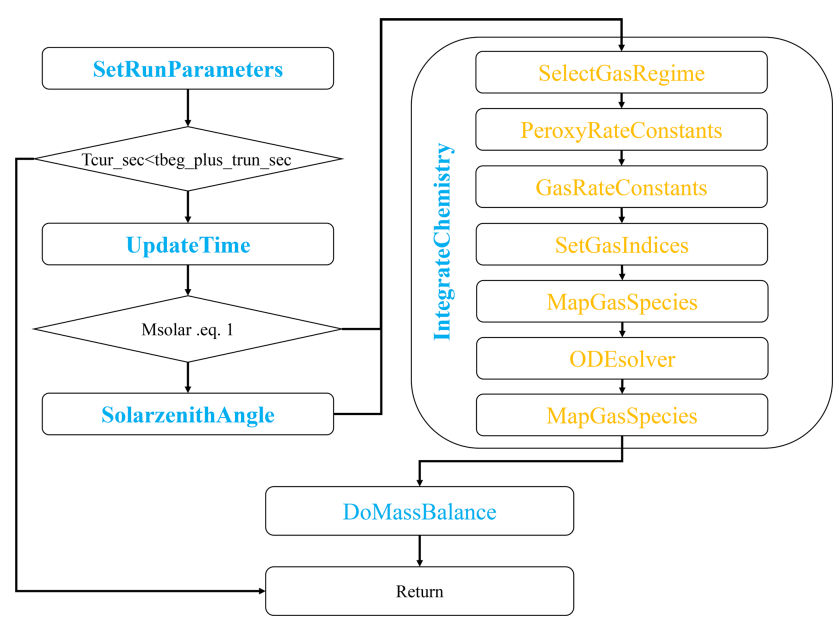

Figure 1. The framework of the CBM-Z gas-phase chemistry module. The functions in the yellow font represent the inner function of IntegrateChemistry.

Table 1. The possible values of iregime and the combination of chemical schemes.

\begin{tabular}{lrrrrrr}
\hline iregime & 1 & 2 & 3 & 4 & 5 & 6 \\
\hline & COM & COM & COM & COM & COM & COM \\
Sub- & HET & HET & HET & HET & HET & HET \\
schemes & & URB & URB & & URB & URB \\
& & & BIO & & & BIO \\
& & & & MAR & MAR & MAR \\
\hline
\end{tabular}

Fortunately, contiguous model grid boxes may have similar chemical processes in air quality simulations, which provides the opportunity to integrate the grid boxes with similar or the same chemical processes to implement vectorization to calculate the processes of multiple grid boxes simultaneously. The following section introduces the details about integrating the chemistry sub-schemes to implement the vectorization.

\subsection{Algorithm description}

The new generation Intel CPU (e.g., Skylake) and Intel MIC chips are equipped with the AVX-512 (AVX - Advanced Vector Extensions) or more advanced vectorization instructions, which support a maximum of 8 double-precision and 16 single-precision operations with 512 bit wide vector registers. It is critical to peak performance of the next-generation CPUs and MICs to fully reach the potential of the AVX512 (Mielikainen et al., 2014). As mentioned in Sect. 2.1, automatic vectorization using a compiler is impeded by the features of CBM-Z, and the common manual measures including constructing loops, avoiding the loop-data dependence, and aligning the data with directives are needed to further vectorize CBM-Z. On the other hand, to implement the vectorization of the module, the general design allowed 
the CBM-Z module to handle multiple grid boxes in one citing cycle, and the functions in CBM- $Z$ were reconstructed by adding a regular loop for these grid boxes. Subsequently, these loops can be vectorized to implement the fine-grained parallelization on a VPU.

All of the model grid boxes are distributed to multiple cores using a message passing interface (MPI) and OpenMP, which is a type of coarse-grain parallelization. Our goal is to implement fine-grained parallelization based on the SIMD, and the grid boxes that are distributed to a specific processor operate in parallel using the VPUs on each core. As shown in Fig. 2, the calling method of the CBM-Z module changes from calculating one model grid box calculation at a time to multiple model grid boxes at the same time. The step length (VLEN in Fig. 2) of the loops represents the number of the grid boxes operated simultaneously, and it is determined by the length of the vector register. The VLEN was set to 16 since the 512 bit wide vector of the AVX-512 can support 16 single-precision operations at the same time. Using this framework, the functions in CBM-Z construct an extra loop to manage the point number dimension, and the corresponding variables require an extra dimension to store the information of multiple grid boxes. Using the structure with an extra loop, it was easier to implement vectorization. Meanwhile, to avoid multiple remaining points which cannot satisfy the VLEN, we set a common variable array, pmask (VLEN) as shown in Fig. 2, to store the availability label of the model grid boxes. When the number of remaining grid boxes did not reach VLEN, the corresponding pmask value of excessive grid boxes was set to "false" to mask these grid boxes in the calculation. Furthermore, the latitude and longitude dimension loops were merged, from nested loops to a single loop, to reduce the number of unavailable points as shown in Fig. 2. Achieving such a large-scale vectorization also requires load balancing of the calculation processes, but the calculation branches in CBM-Z are an obstacle to this. Therefore, the branches in CBM-Z should be taken into consideration in constructing the loops, especially the chemical schemes chosen in Table 1. As mentioned in Sect. 2.1, the contiguous model grid boxes may have similar chemical processes in the atmosphere. This provides an opportunity to integrate the sub-schemes by masking the heterogeneous model grid boxes, and this type of masking operation can be used in the functions GasRateConstants and ODEsolver (Fig. 1). Figure 3 shows the flowchart for masking the model grid boxes to satisfy the vectorization of the grid array. A set of grid boxes with the number of VLEN (16 in this study) would perform the operation simultaneously, and the variable pmask signed the valid grid boxes. Meanwhile, the variable iregime described in Table 1 and representing the combination of sub-schemes, is used to determine whether the model grid must perform the subsequent operation or not. The grid boxes with the same property or calculation are kept by setting the variable bmask to "true". The COM and HET schemes are common for all grid boxes, and the mask operation for COM and HET schemes only determines the availability of the grid boxes. As shown in Fig. 3, for the URB, BIO, and MAR schemes, the iregime value and pmask are both used to filter the heterogeneous grid boxes and the bmask stores the results. To improve the efficiency of vectorization, the bmask does not prevent the calculation of heterogeneous grid boxes but prevents the calculation results from being copied back to the return value. Thus, all computations are performed on all grid boxes, but only the results of the valid grid boxes are returned. This improves the utilization of data as well as the efficiency of vectorization. Because of the independence of the grid boxes, the computation process of VLEN arrays is independent and satisfies the requirement of vectorization, and the corresponding directives were added to declare the independence of the arrays and force the compiler to perform the data alignment and vectorization after the reconstruction of the code. Overall, by constructing the loops, the computations of the independent grid boxes were integrated with the fine-level parallel implementation through the SIMD. In addition, the efficiency of such algorithms is linearly improved with the development of the width of the vector in the VPU.

\section{Test results}

The validation and evaluation of the improvement of the new method were conducted using the box model of CBM-Z as well as a regional CTM named Nested Air Quality Prediction Model System with the optimized CBM-Z scheme. We tested the theoretical performance of vectorization by using the box model, and the CTM tests illustrate its potential in three dimensions with varying chemical regimes.

\subsection{Benchmark platform description}

The computation cluster for tests was provided by the Institute of Atmospheric Physic (IAP), Chinese Academy of Sciences (CAS). The CPU and KNL platforms were used for testing the code. The CPU platforms in this study include two generation CPUs, two-socket CPU nodes with Broadwell architecture $2.4 \mathrm{GHz}$ 14-core Intel Xeon E5-2680 V4 processors, and two-socket CPU nodes with $2.6 \mathrm{GHz}$ Skylake architecture 14-core Intel Xeon Gold 6132. To the vector instructions, the previous generation of Broadwell adopted the AVX-2 vector instructions and the new generation used the AVX-512 vector instructions. The AVX-512 and AVX2 instructions support 16 and 8 single-precision floatingpoint calculations simultaneously, respectively. Comparing the two generation CPUs helped to present the potential of new MP CBM-Z to fully use the development of hardware. The KNL node contained one $1.4 \mathrm{GHz} 68$-core Intel Xeon Phi 7250 processor, which also adopted the AVX-512 vector instructions. The operating system was Cent OS Linux 7.4.1708 for all platforms. The code was all compiled us- 
(a)
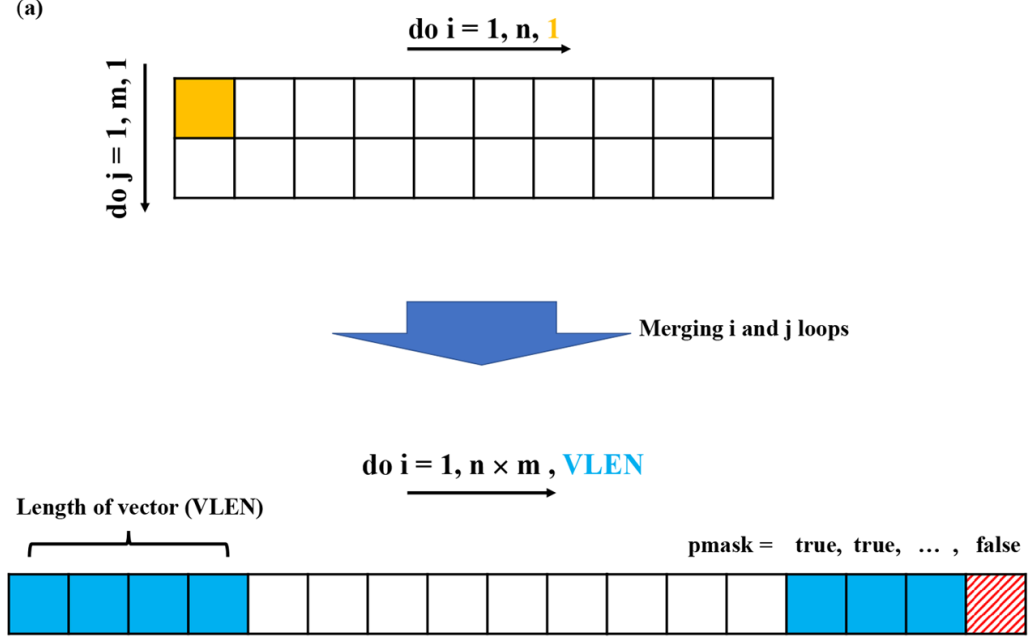

(b)

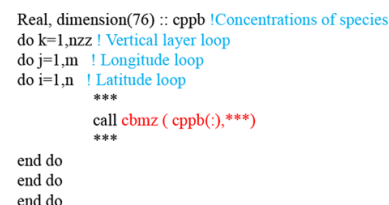

(c)

Integer, parameter $: . \mathrm{VLEN}=16$ ! The length of vector for AVX 5 1 Logic, dimension(VLEN) :: pmask !Mask Array do $\mathrm{k}=1, \mathrm{nzz}$ ! vertical laver loop norids $=n^{*} m !$ Total number of grids do $\mathrm{i}=\mathbf{1}$, ngrids, VLEN ! Merged loops of longitude and latitude grids ilen $=\min ($ ngrids-i, VLEN) $!$ Decide the length of this vector pmask $=$ False !exclude the invalid grids do $\mathrm{j}=1$, ilen $\operatorname{pmask}(j)=$ True end do $\underset{* * *}{\operatorname{call} \operatorname{cbmz}(\operatorname{cppb}(,,), * * *)}$ end do

Figure 2. A schematic diagram of the changes in the calling method of CBM-Z. The calling method of the CBM-Z module changes from calculating one model grid calculation at a time to multiple model grid boxes at the same time. The VLEN represents the number of points operated simultaneously, which is determined by the length of the register in the vector processing unit (VPU). The $i$ and $j$ loops, equaling latitude and longitude loops, were merged to construct one vector to reduce the number of unfilled vectors. Panel (b) and (c) illustrate the sample code before and after integrating grid boxes.

(a)

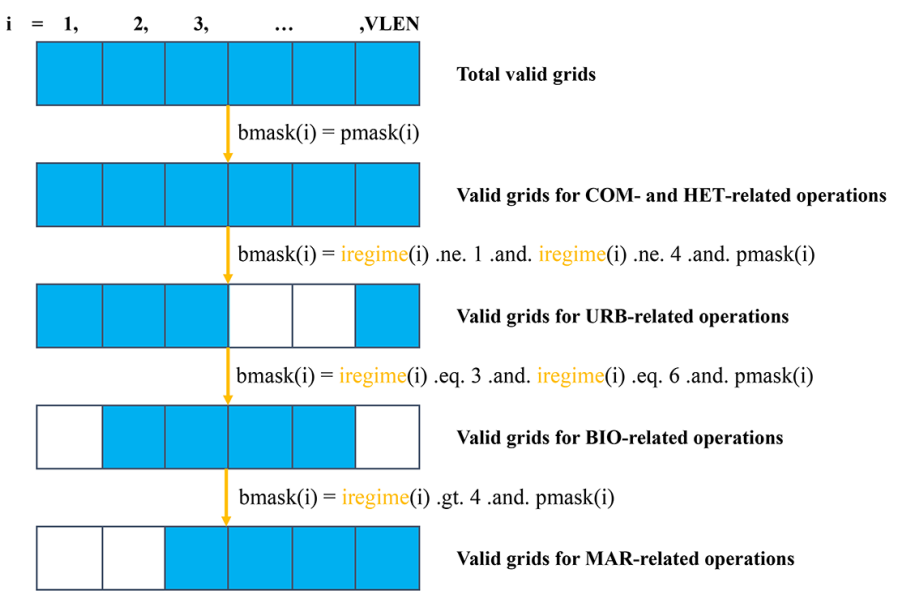

(b)

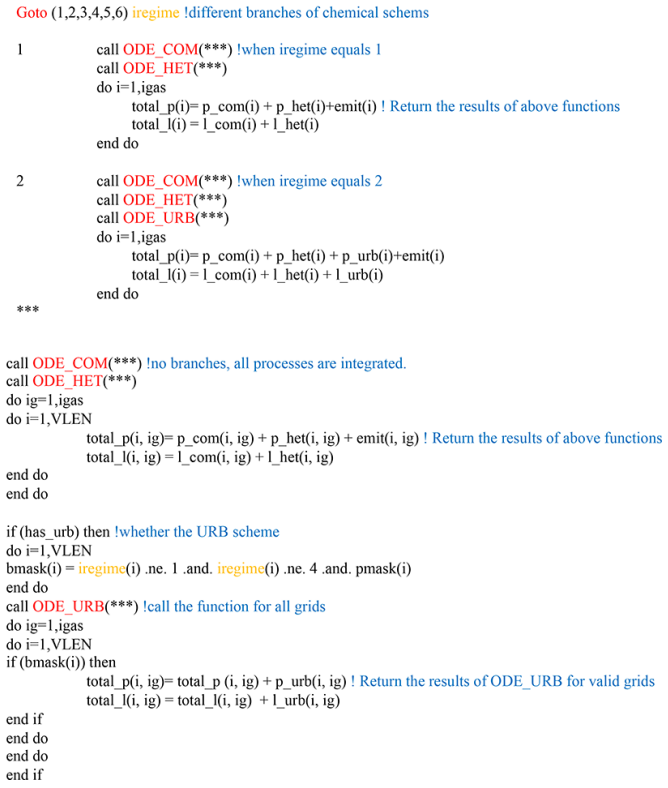

Figure 3. The flowchart (a) shows the way to mask the heterogeneous girds to integrate grid boxes to perform the vectorization operations according to the iregime values. Panels (b) and (c) illustrate the sample code before and after integrating grid boxes. In panel (b), iregime leads different calling processes; in panel (c), the calling processes are integrated into one flow, and the functions are called for all grid boxes but only the values of valid grid boxes are returned.

ing the Intel FORTRAN Compiler 2017 update 4, and the compile flags for vectorization and float-pointing accuracy of the CBM-Z module and the NAQPMS are shown in Tables 2 and 3 , respectively. The corresponding flags for vectorization (e.g., -xCORE-AVX2, -xCOMMON-AVX512, -xMIC$A V X 512$, -align array64byte) were adopted for MP CBM-Z.
We also tested the code using diverse options for the compile flag -fp-model, which controls the balance between accuracy and performance of floating-point calculations, to investigate its impact on code. We mainly consider the two options of $f p$-model precise and - fp-model fast $=1$. The fast $=1$ is the default option when -fp-model flag is not selected. Compared 
with the option precise, the fast=l improves the computational performance but reduces the accuracy of the floatingpoint calculations. Using precise is a safer option and forces the compiler to avoid the vectorization of some calculations to improve accuracy. We compare the results of the two options, including the outputs and the performance of models, to investigate its impact and discuss a suitable choice of compile flag.

\subsection{Box model test}

The box model of MP CBM-Z was used to validate the model outputs and investigate the ideal parallel performance of the single module. We also tested the results using different parallelization techniques, e.g., MPI and OpenMP. Each test was repeated 10 times to reduce the impact from any platform variability.

\subsubsection{Test case description}

There are two cases that were used for the CBM-Z box model. One was a $10 \mathrm{~h}$ single grid box case with all species to validate the outputs of the model, and the other was a $1 \mathrm{~h}$ simulation with $160 \times 148 \times 20$ grid boxes to test the performance of the module under a more realistic scenario. The initial values for the single grid box are shown in Table S1 in the Supplement. The meteorological conditions were constant and emissions were set to zero to test the error of the algorithms. The time step was $5 \mathrm{~s}$ for the two cases. For validation purposes, output every 5 min was used, while the computational performance test did not include the output function to eliminate any impact from input-output (I/O). The different compiling flags for the precision of floating-point calculations are presented in Table 2. We test the baseline and the optimized model on two different platforms of CPU and KNL, and the computational time was counted using the system_clock function.

\subsubsection{Box model validation}

We evaluate the chemical species including ozone $\left(\mathrm{O}_{3}\right)$, nitrogen dioxide $\left(\mathrm{NO}_{2}\right)$, nitrogen monoxide (NO), hydrogen peroxide $\left(\mathrm{H}_{2} \mathrm{O}_{2}\right)$, sulfur dioxide $\left(\mathrm{SO}_{2}\right)$, sulfuric acid $\left(\mathrm{H}_{2} \mathrm{SO}_{4}\right)$, hydroxyl $(\mathrm{OH})$ radical, hydroperoxyl $\left(\mathrm{HO}_{2}\right)$ radi$\mathrm{cal}$, and alkyl peroxy $\left(\mathrm{RO}_{2}\right)$ radical. These species are important for tropospheric gas-phase chemistry and sulfate aerosol formation and hence suitable for validating whether the optimization significantly changed the simulated results or not. Figure 4 shows the time series of the simulated concentrations of the species by the baseline (base) and the optimized (opt) model with the precise and fast $=1$ compile flags. The results with the baseline code with precise compile flag is the benchmark, and there is no difference between the results from the baseline and optimized code with the same precise compile flag. The precise compile flag is a relatively safe compile flag and prohibits optimizations that can affect the accuracy. The fast=l compile flag can lead to errors even with the same code, but the relative error (RE) of the baseline code with fast $=1$ compile flag relative to the benchmark is extremely small $(<0.0002 \%)$. As shown in Fig. 4, with the optimized code, the fast $=1$ compile flag results in a maximum RE of $0.025 \%$ for $\mathrm{NO}$ and $\mathrm{NO}_{2}$ at the end of the simulation. We find that the error caused by the fast $=1$ compile flag did not become obvious for species with low concentrations of $\mathrm{OH}$ and $\mathrm{RO}_{2}$. We will further discuss the impact of the fast $=1$ compile flag in Sect. 3.3.2 in the context of CTM simulations.

\subsubsection{Box model computational performance}

The case with $160 \times 148 \times 20$ grid boxes was used to test the computational performance. Both the baseline and the optimized version of CBM-Z contained the same 76 species. The computational time of the baseline version on a single core of E5-2680 V4 CPU with the precise compile flag was considered as the benchmark time. The tests were done with two generations of CPUs and KNL.

The option of -fp-model could directly affect the performance. As shown in Table 4, the benchmark performance was $1014.67 \mathrm{~s}$ on the E5-2680 V4 platform. By using the new platform with Intel Gold 6132, the baseline version code achieves $1.52 \times$ speedup with the precise compile flag. The fast $=1$ compile flag leads to $1.28 \times$ and $2.04 \times$ speedups for the baseline code on both CPUs. Meanwhile, updating the CPU enables the original CBM-Z module to gain a speedup of about 1.52 and 1.59 with precise and fast $=1$ compile flags, respectively.

The MP CBM-Z module shows good performance on both CPUs. On the E5-2680 V4 CPU with Broadwell architecture, the optimized code with two different compile flags consumed 581.14 and $153.32 \mathrm{~s}$, respectively; meanwhile, the speedups reach $1.75 \times$ and $6.62 \times$ compared with the benchmark performance. In regard to the Intel Gold 6132 platform, the optimized version CBM-Z consumed 352.00 and $55.42 \mathrm{~s}$ with precise and fast $=1$ compile flags, respectively. Compared with the benchmark time, the speedups reach $2.88 \times$ and $18.31 \times$. By using the same fast $=1$ compile option, the MP CBM-Z shows $5.16 \times$ and $8.97 \times$ speedups on two generations of CPU compared with the original CBM-Z code.

The results also illustrate that the optimized code could better utilize the updating of cores through good vectorization ability compared with the baseline code. Comparing the performance of the optimized code, we find that updating the CPU could lead to about 1.65 times and 2.76 times acceleration with precise and fast $=1$ compile flags, respectively, which is higher than the $1.5 \times$ speedup gained with the baseline code.

Compile flags largely affect the code performance on KNL. On the Xeon Phi 7250 platform, the optimized code took $3454.90 \mathrm{~s}$ with the precise compile flag since the majority of vectorizations were forbidden, and it is even slower 
Table 2. Compile flags of the different versions of CBM-Z.

\begin{tabular}{|c|c|c|c|}
\hline \multirow[t]{2}{*}{ Version of CBM-Z } & \multirow[t]{2}{*}{ Processor } & \multicolumn{2}{|c|}{ Intel compiler flags } \\
\hline & & Flags for vectorization & $\begin{array}{l}\text { Flags for } \\
\text { floating-point accuracy }\end{array}$ \\
\hline \multirow{2}{*}{ Baseline CBM-Z } & Xeon E5-2680 V4 & $\begin{array}{l}-x C O R E-A V X 2 \\
-x C O R E-A V X 2\end{array}$ & $\begin{array}{l}-f p \text {-model precise } \\
-f p \text {-model fast }=1\end{array}$ \\
\hline & Xeon Gold 6132 & $\begin{array}{l}-x C O M M O N-A V X 512 \\
-x C O M M O N-A V X 512\end{array}$ & $\begin{array}{l}-f p \text {-model precise } \\
-f p \text {-model fast }=1\end{array}$ \\
\hline \multirow{3}{*}{ MP CBM-Z } & Xeon E5-2680 V4 & $\begin{array}{l}-x C O R E-A V X 2 \\
-x C O R E-A V X 2\end{array}$ & $\begin{array}{l}-f p \text {-model precise } \\
-f p \text {-model fast }=1\end{array}$ \\
\hline & Xeon Gold 6132 & $\begin{array}{l}-x C O M M O N-A V X 512 \\
-x C O M M O N-A V X 512\end{array}$ & $\begin{array}{l}-f p \text {-model precise } \\
-f p \text {-model fast }=1\end{array}$ \\
\hline & Xeon Phi 7250 & $-x M I C-A V X 512$ & $-f p$-model fast $=1$ \\
\hline
\end{tabular}

Table 3. Compile flags of the different versions of NAQPMS.

\begin{tabular}{|c|c|c|c|}
\hline \multirow[t]{2}{*}{ Version of NAQPMS } & \multirow[t]{2}{*}{ Processor } & \multicolumn{2}{|c|}{ Intel compiler flags } \\
\hline & & Flags for vectorization & $\begin{array}{l}\text { Flags for } \\
\text { floating-point accuracy }\end{array}$ \\
\hline \multirow{2}{*}{ Baseline NAQPMS } & Xeon E5-2680 V4 & $\begin{array}{l}-x C O R E-A V X 2 \\
-x C O R E-A V X 2\end{array}$ & $\begin{array}{l}-f p \text {-model precise } \\
-f p \text {-model fast }=1\end{array}$ \\
\hline & Xeon Gold 6132 & $\begin{array}{l}-x C O M M O N-A V X 512 \\
-x C O M M O N-A V X 512\end{array}$ & $\begin{array}{l}-f p \text {-model precise } \\
-f p \text {-model fast }=1\end{array}$ \\
\hline \multirow{3}{*}{ NAQPMS with MP CBM-Z } & Xeon E5-2680 V4 & $\begin{array}{l}-x C O R E-A V X 2 \\
-x C O R E-A V X 2\end{array}$ & $\begin{array}{l}-f p \text {-model precise } \\
-f p \text {-model fast }=1\end{array}$ \\
\hline & Xeon Gold 6132 & $\begin{array}{l}-x C O M M O N-A V X 512 \\
-x C O M M O N-A V X 512\end{array}$ & $\begin{array}{l}-f p \text {-model precise } \\
-f p \text {-model fast }=1\end{array}$ \\
\hline & Xeon Phi 7250 & $-x M I C-A V X 512$ & $-f p-$ model fast $=1$ \\
\hline
\end{tabular}

than the benchmark performance; it only took 214.09 seconds and obtained a speedup of $4.74 \times$ with the fast=1 compile flag. Compared with the baseline CBM-Z with the fast=1 flag on Intel Xeon E5-2680 V4, KNL gains a 3.69× speedup with the MP CBM-Z.

In addition, the baseline and optimized code with fast $=1$ were also analyzed by using the high-performance computing (HPC) performance characterization from the Intel VTune tools on the CPU platform. On the Intel Gold 6132 platform, the single-precision giga-floating point operations calculated per second (GFLOPS) increased from 4.81 to 21.37 compared with the original CBM-Z module, and the vector capacity usage improved from $14.3 \%$ in the baseline CBM-Z to $89.4 \%$ in the MP CBM-Z, which implies that the majority of floating-point instructions in CBM-Z were vectorized.

We also tested the parallel version of the MP CBM-Z by compiling with the fast=1 option and with MPI and OpenMP separately. We evaluated the speedups based on the performance of the baseline CBM-Z on the Intel Xeon E52680 V4 platform with fast $=1$ option. The results are shown in Table 5. The MPI and OpenMP version of CBM-Z had a $104.63 \times$ speedup and $101.02 \times$ speedup on the Intel Xeon E5-2680 V4 platform. On the new Intel Xeon Gold 6132, the MP CBM-Z got a speedup of $198.50 \times$ and $194.60 \times$ with MPI and OpenMP. For the KNL, the speedup reached $175.23 \times$ by using MPI and $167.45 \times$ by using OpenMP, which was approximately $40 \%$ faster than those on the twosocket Broadwell platform with AVX2 vectorization instruction and about $13 \%-16 \%$ slower than those on the twosocket Skylake platform with the same AVX512 vectorization instruction. The combination of the fine-grain vectorization and the coarse-grain parallelization of OpenMP/MPI results in a significant performance improvement on the new generation processors. The enhancement of the vectorization performance may be the key to fully using the new generation processors equipped with advanced and wider vectors 
(a)
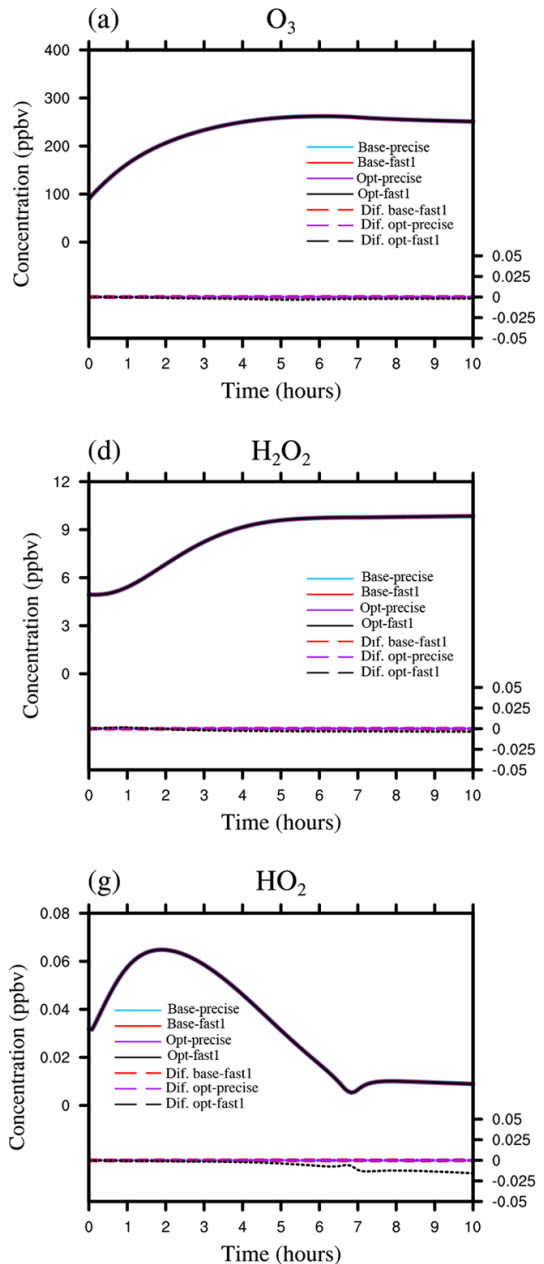

(b)

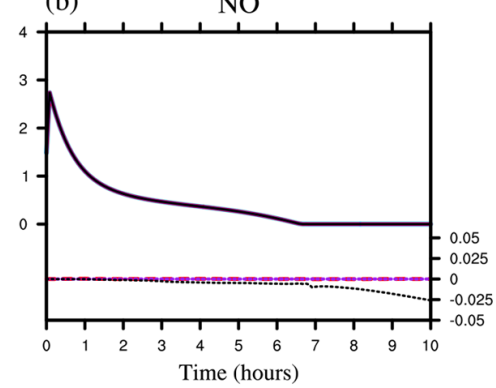

(e)
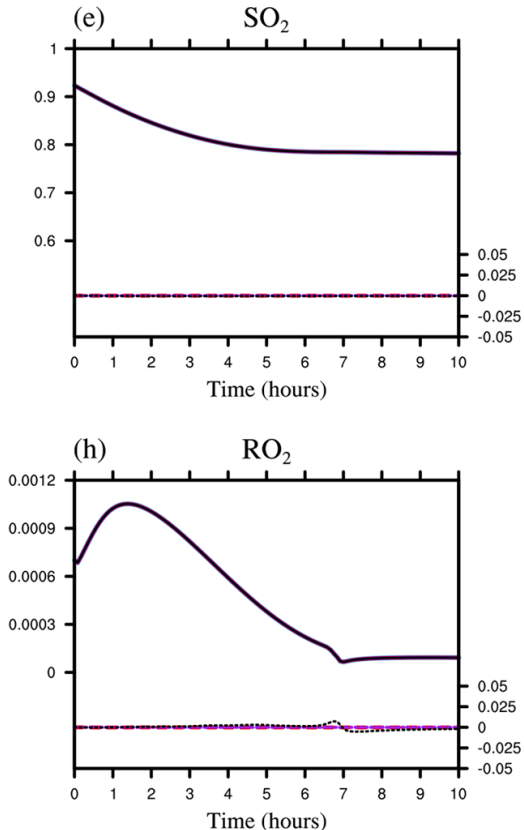

(c) $\mathrm{NO}_{2}$
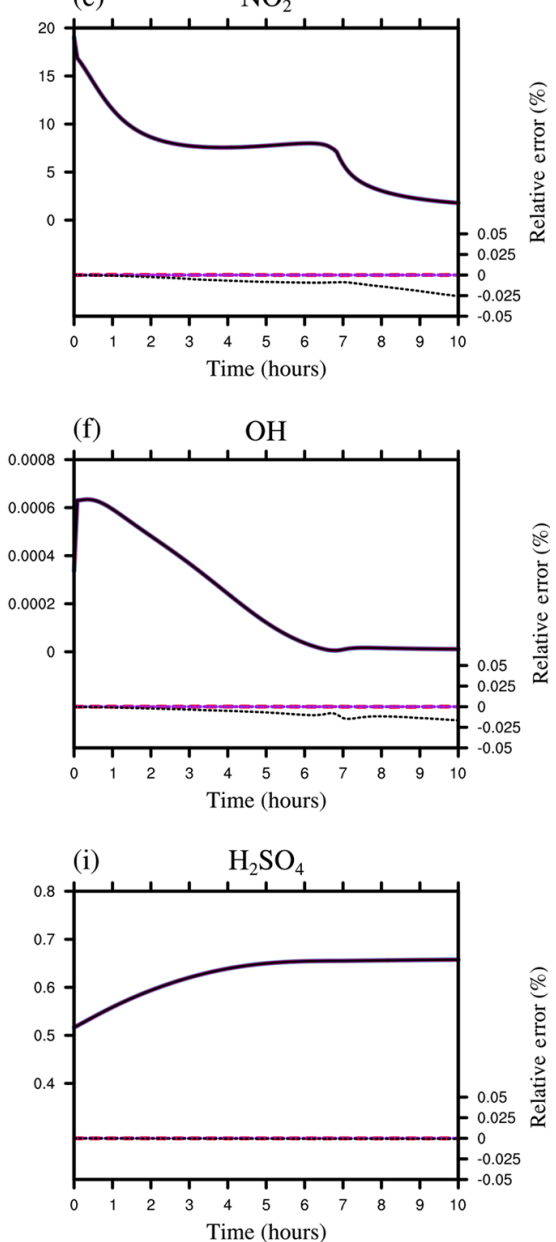

Figure 4. Comparison of the time-series concentrations of $\mathrm{O}_{3}, \mathrm{NO}, \mathrm{NO}_{2}, \mathrm{H}_{2} \mathrm{O}_{2}, \mathrm{SO}_{2}, \mathrm{OH}, \mathrm{HO}_{2}, \mathrm{RO}_{2}$, and $\mathrm{H}_{2} \mathrm{SO}_{4}(\mathbf{a}-\mathbf{i})$ from the baseline and optimized CBM-Z simulation with diverse - $f$-model options. The simulation results by the baseline code with the $-f p$-model precise compile flag was as the benchmark. The solid lines show the time-series concentrations of the species from different experiments and the dashed lines showed the relative errors (RE) of simulated concentrations between the benchmark and the results by other combinations of the code and -fp-model options.

Table 4. The performance tests of the baseline and optimized code on different CPUs and KNL platforms with one physical cores. The unit of the wall times for the tests is seconds (s).

\begin{tabular}{llllrr}
\hline & Processor & Vector instruction & -fp-model & Wall time & Speedup \\
\hline \multirow{3}{*}{ Baseline CBM-Z } & \multirow{2}{*}{ Xeon E5-2680 V4 } & \multirow{2}{*}{ AVX2 } & precise & 1014.67 & 1.00 \\
& & & fast=1 & 792.03 & 1.28 \\
\cline { 2 - 6 } & \multirow{2}{*}{ Xeon Gold 6132 } & \multirow{2}{*}{ AVX512 } & precise & 665.44 & 1.52 \\
& & fast=1 & 497.64 & 2.04 \\
\hline \multirow{2}{*}{ Xeon E5-2680 V4 } & \multirow{2}{*}{ AVX2 } & precise & 581.14 & 1.75 \\
& \multirow{2}{*}{ Xeon Gold 6132 } & \multirow{2}{*}{ AVX512 } & fast=1 & 153.32 & 6.62 \\
\cline { 2 - 6 } & & & precise & 352.00 & 2.88 \\
& \multirow{2}{*}{ Xeon Phi 7250 } & \multirow{2}{*}{ AVX512 } & fast=1 & 55.42 & 18.31 \\
\hline \multirow{2}{*}{} & & precise & 3454.90 & 0.29 \\
& & & fast=1 & 214.09 & 4.74 \\
\hline
\end{tabular}


and can be important in making full use of the new MIC architecture processors such as KNL.

\subsection{CTM test}

The regional CTM, the NAQPMS (Wang et al., 2001; ZiFa et al., 2006), was used to test the MP CBM-Z module under more realistic conditions. The following subsections will describe the CTM test case and will present results from the scientific validation and its computational performance.

\subsubsection{CTM test case description}

The NAQPMS is a regional CTM developed by IAP, CAS (Li et al., 2011, 2013), and has been widely used in air quality research (Wang et al., 2018) and routine air quality forecasting (Wu et al., 2010; Chen et al., 2013). NAQPMS involves all essential processes including diffusion, advection, dry and wet deposition, and multiphase chemistry reactions. More details can be found in Li et al. (2013). In a similar way to the box model test case, the NAQPMS with the baseline and optimized CBM-Z modules were compiled with various compile flags as shown in Table 3.

The test case is a $72 \mathrm{~h}$ simulation covering the East Asia region. The horizontal resolution is $15 \mathrm{~km}$ with $339 \times 432$ grid boxes. The model adopted 20 vertical layers. The meteorological fields driving the NAQPMS were provided by the Weather Research and Forecasting (WRF) model (Skamarock et al., 2008). The anthropogenic emission inventory was from the Hemispheric Transport of Air Pollution (HTAP) V2 and the biogenic emission inventory was provided by results from Sindelarova et al. (2014) using the Model of Emissions of Gases and Aerosols from Nature (MEGAN) (Guenther et al., 2006, 2012). The simulation started at 00:00 UTC, 17 August 2015, and ended at 00:00 UTC, 20 August 2015. We only used one node for testing to exclude the interference of network communication. Each experiment was repeated five times and the performance was assessed on the basis of the average value.

\subsubsection{CTM validation}

We chose four major gas pollutants, i.e., $\mathrm{NO}_{2}, \mathrm{O}_{3}, \mathrm{SO}_{2}$, and $\mathrm{CO}$, after $72 \mathrm{~h}$ integration to evaluate the optimized code. The simulation results of the baseline NAQPMS code compiled by the precise flag were as the benchmark results, and we mainly compared the simulation results of the baseline NAQPMS code with the fast=l flag and the optimized NAQPMS with precise and fast $=1$.

Figures 5 and 6 present the spatial distributions of $\mathrm{NO}_{2}$, $\mathrm{O}_{3}, \mathrm{SO}_{2}$, and $\mathrm{CO}$ as well as the absolute errors (AEs) of their concentrations from other experiments relative to the baseline. We find that all model results show the same spatial distribution of pollutants. In general, for $\mathrm{NO}_{2}, \mathrm{O}_{3}$, and $\mathrm{SO}_{2}$, the AEs in the majority of grid boxes are in the range of $\pm 0.02 \mathrm{ppbv}$ for the three experiments; for $\mathrm{CO}$, the AEs of baseline and optimized NAQPMS with the same fast $=1$ are outside that range, showing more obvious AEs than that of other species.

The precise option enables the results of the two versions to be more consistent. Figure 7 shows the distribution of AEs and relative error (REs) for four species in the near-surface model layer. For the majority of points, the AEs and REs are in a relatively small range. However, some points show exceptional and obvious errors. The maximum AEs for $\mathrm{NO}_{2}$, $\mathrm{O}_{3}, \mathrm{SO}_{2}$, and $\mathrm{CO}$ are $0.166,0.197,0.001$, and $0.03 \mathrm{ppb}$ over the whole map after $72 \mathrm{~h}$ of integration, and the fast $=1$ option shows more obvious errors for both versions. For the baseline NAQPMS code, using fast $=1$ leads to maximum AEs of $0.23,4.5,0.17$, and $2.6 \mathrm{ppbv}$ for $\mathrm{NO}_{2}, \mathrm{O}_{3}, \mathrm{SO}_{2}$, and $\mathrm{CO}$, respectively. To NAQPMS with the MP CBM-Z, using the fast $=1$ option leads to maximum $0.13,0.93,0.76$, and 0.64 ppbv AEs for $\mathrm{NO}_{2}, \mathrm{O}_{3}, \mathrm{SO}_{2}$, and $\mathrm{CO}$ over the whole domain, which is better than the baseline NAQPMS.

In addition to considering the accuracy mentioned above, the impact of the -fp-model option on performance should be considered. In some pragmatic applications like routine air quality prediction, it is reasonable to sacrifice accuracy to gain computational performance. Conversely, applications like long-term climate simulations, choosing safer compile flags, or adopting double-precision for calculations to avoid accumulation of errors.

\subsubsection{CTM computational performance}

The performance of the baseline NAQPMS with precise was the benchmark for comparison with other tests. As shown in Table 6, in the original version of NAQPMS, the CBM$\mathrm{Z}$ module accounts for $72.26 \%$ of the wall-clock time for the whole simulation. Changing the compile option of $-f p$ model to improve performance by sacrificing accuracy leads to $1.34 \times$ and $1.25 \times$ speedups for the module CBM-Z and the whole model on the Intel Xeon E5-2680 platform, respectively. By updating the CPU from Intel Xeon E5-2680 to Intel Xeon Gold 6132, the module CBM-Z and whole model gain $1.28 \times$ and $1.29 \times$ speedups, respectively. The speedups improve to $1.68 \times$ and $1.58 \times$ for CBM-Z and the whole model, respectively, by using the fast $=1$ compile flag on the Xeon Gold 6132 platform. The benefit from updating hardware is limited with the baseline code and supports the need for optimizing code to adapt to the new hardware features.

The computational performance of the gas-phase chemistry module and the NAQPMS are largely improved after adopting the MP CBM-Z, as described in this paper. As shown in Table 6, the CBM-Z model and the whole NAQPMS shows speedups of $1.59 \times$ and $1.40 \times$ on the old Xeon E5-2680 platform with the same precise compile flag, and the speedups are improved to $4.45 \times$ and $2.44 \times$ by using the fast=1 compile flag. With the same fast=1 flag, the MP CBM-Z showed 3.32 and 1.96 times acceleration compared with the baseline CBM-Z for the gas-phase chemistry 

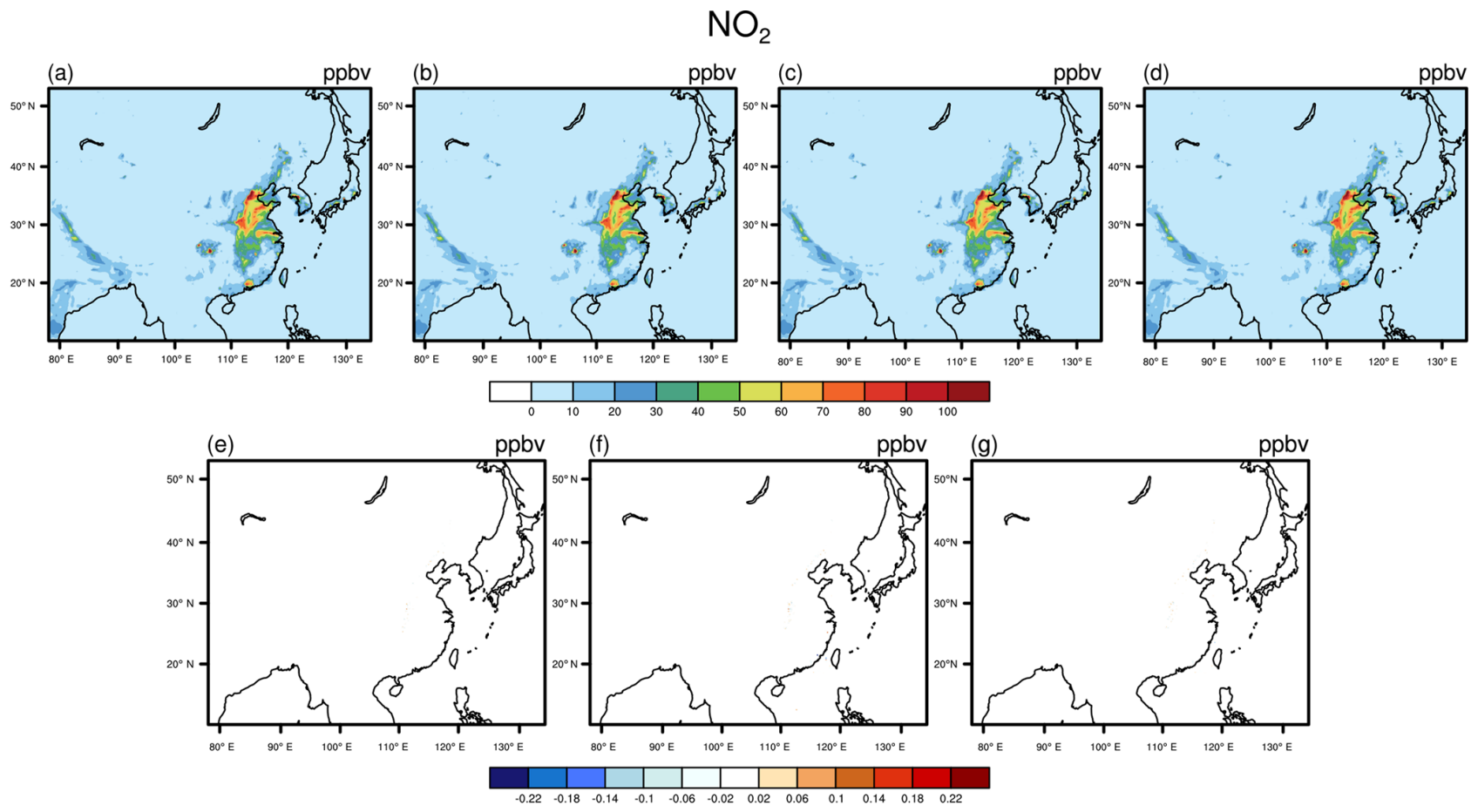

\section{$\mathrm{O}_{3}$}
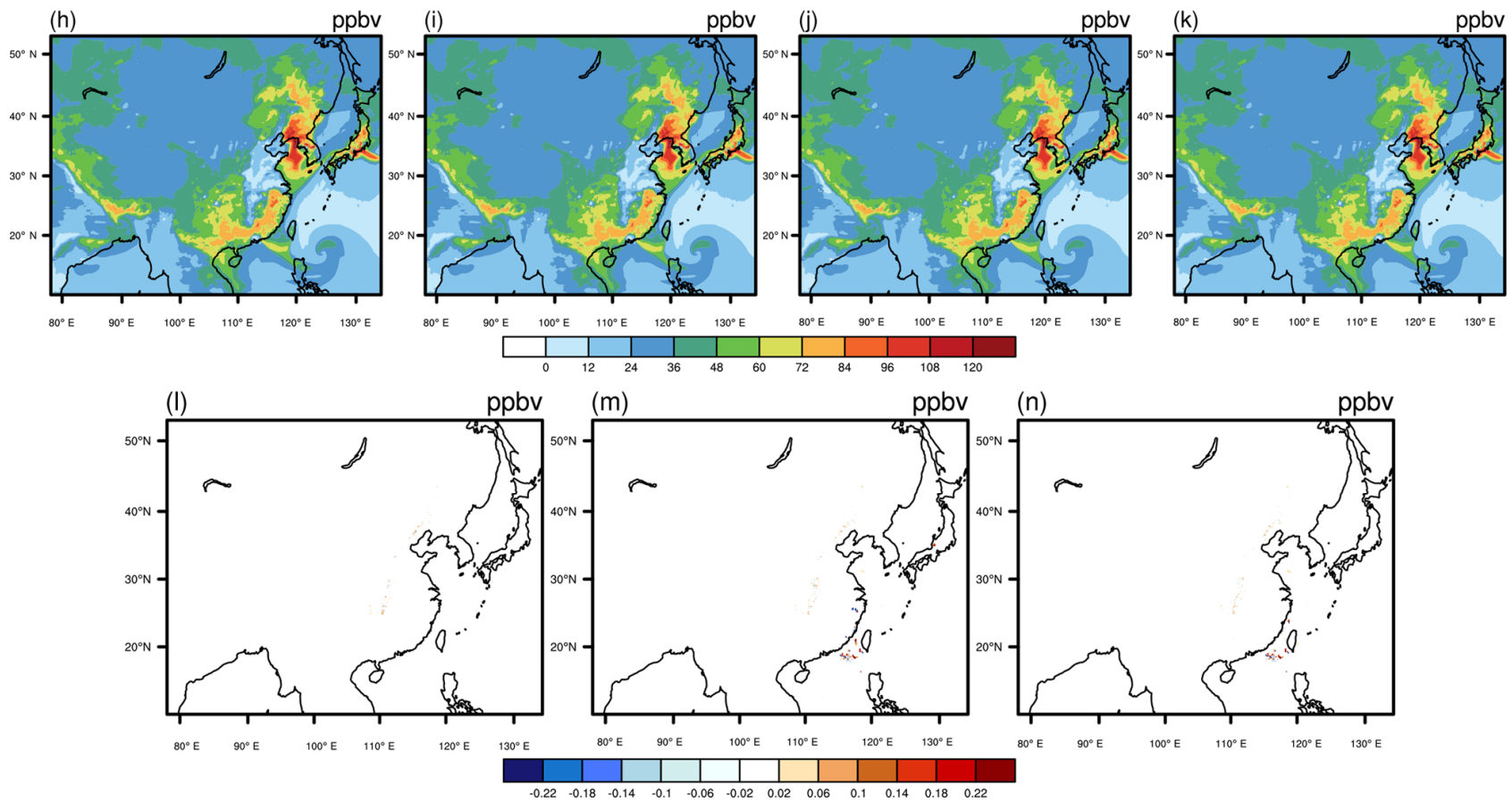

Figure 5. $\mathrm{NO}_{2}$ and $\mathrm{O}_{3}$ concentrations outputted by baseline and optimized code with different accuracy compile flags. Panels (a) and (h) are from baselines code compiled by the precise option, which are treated as benchmark for comparison. Panels (b) and (i) are from optimized code compiled by the precise option. Panels (c) and (j) are from baseline codes compiled by the fast=1 flag. Panels (d) and (k) are from optimized code compiled by the fast=l flag. Panels (e-g) and (l-m) are the output concentration differences of optimized code (precise), baseline code $(f a s t=1)$, and optimized code $(f a s t=1)$ compared with baseline code (precise). 

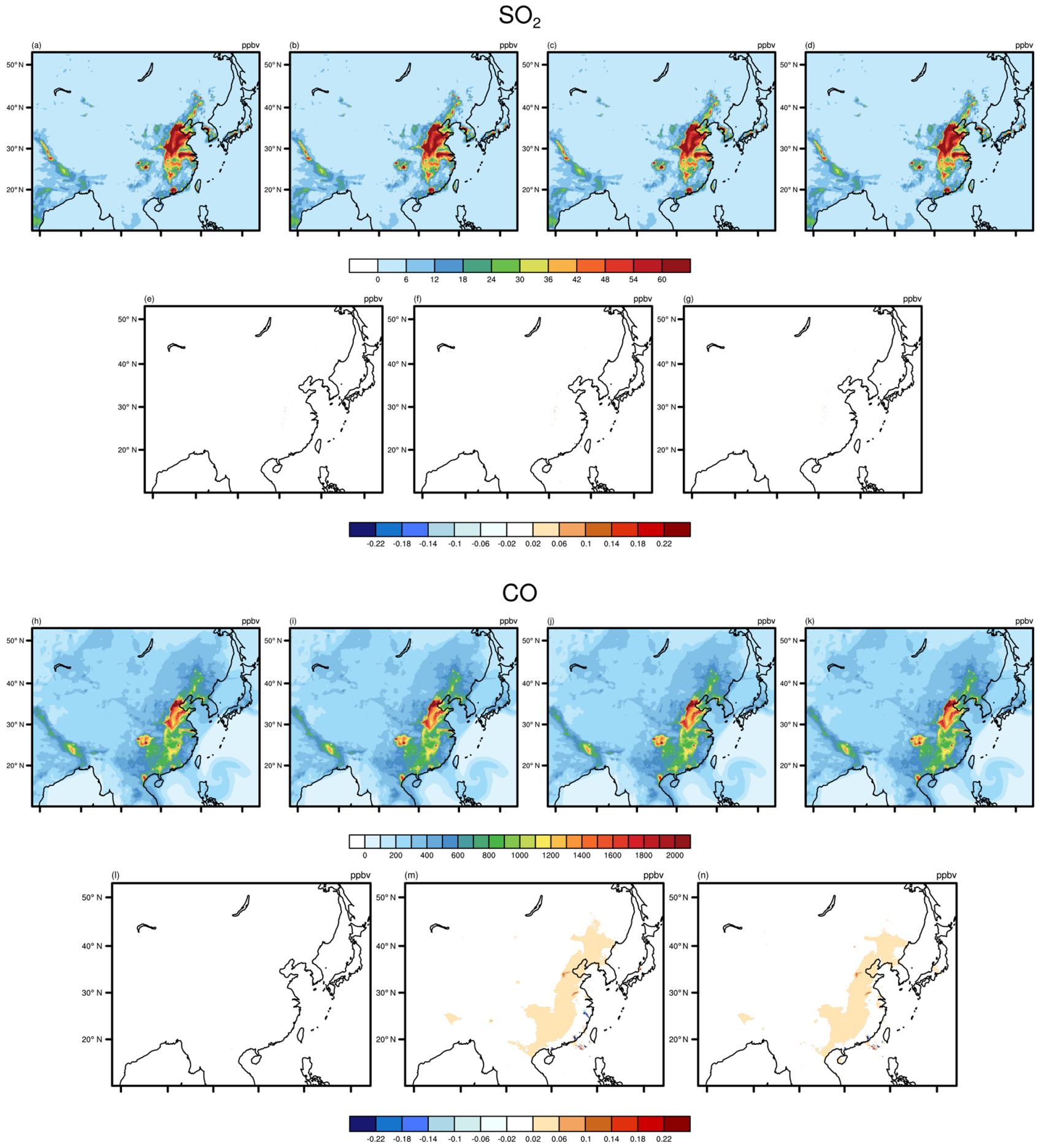

Figure 6. $\mathrm{SO}_{2}$ and $\mathrm{CO}$ concentrations outputted by baseline and optimized code with different accuracy compile flags. Panels (a) and (h) are from baselines code compiled by the precise option, which are treated as benchmark for comparison. Panels (b) and (i) are from optimized code compiled by the precise option. Panels (c) and (j) are from baseline codes compiled by the fast=l flag. Panels (d) and (k) are from optimized code compiled by the $f a s t=1$ flag. Panels (e-g) and (l-m) are the output concentration differences of optimized code (precise), baseline code ( $f a s t=1)$, and optimized code (fast $=1$ ) compared with baseline code (precise). 
Table 5. The performance tests of the optimized code on different CPUs and KNL platforms with MPI and OpenMP. The unit of the wall times for the tests is seconds (s).

\begin{tabular}{|c|c|c|c|c|c|}
\hline \multirow{11}{*}{$\begin{array}{l}\sum_{0}^{N} \\
\sum \\
\sum\end{array}$} & \multicolumn{5}{|l|}{ Single core test } \\
\hline & Processor & $\begin{array}{l}\text { Vector } \\
\text { instruction }\end{array}$ & $\begin{array}{l}\text { Number } \\
\text { of cores }\end{array}$ & $\begin{array}{l}\text { Wall } \\
\text { time }\end{array}$ & Speedup \\
\hline & Xeon E5-2680 V4 & AVX2 & 1 & 792.03 & 1.00 \\
\hline & \multicolumn{5}{|c|}{ MPI with vectorization } \\
\hline & Xeon E5-2680 V4 & AVX2 & 28 & 7.57 & 104.63 \\
\hline & Xeon Gold 6132 & AVX512 & 28 & 3.99 & 198.50 \\
\hline & Xeon Phi 7250 & AVX512 & 68 & 4.52 & 175.23 \\
\hline & \multicolumn{5}{|c|}{ OpenMP with vectorization } \\
\hline & Xeon E5-2680 V4 & AVX2 & 28 & 7.84 & 101.02 \\
\hline & Xeon Gold 6132 & AVX512 & 28 & 4.07 & 194.60 \\
\hline & Xeon Phi 7250 & AVX512 & 68 & 4.73 & 167.45 \\
\hline
\end{tabular}

$\mathrm{NO}_{2}$

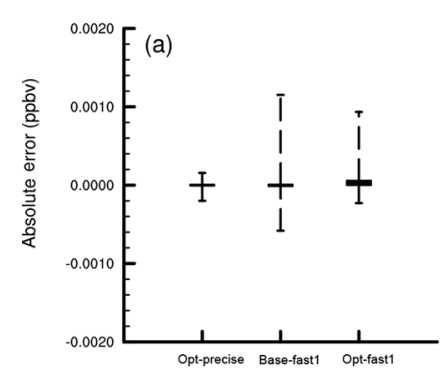

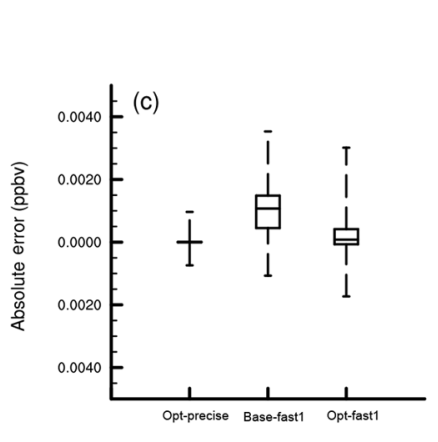

$\mathrm{O}_{3}$
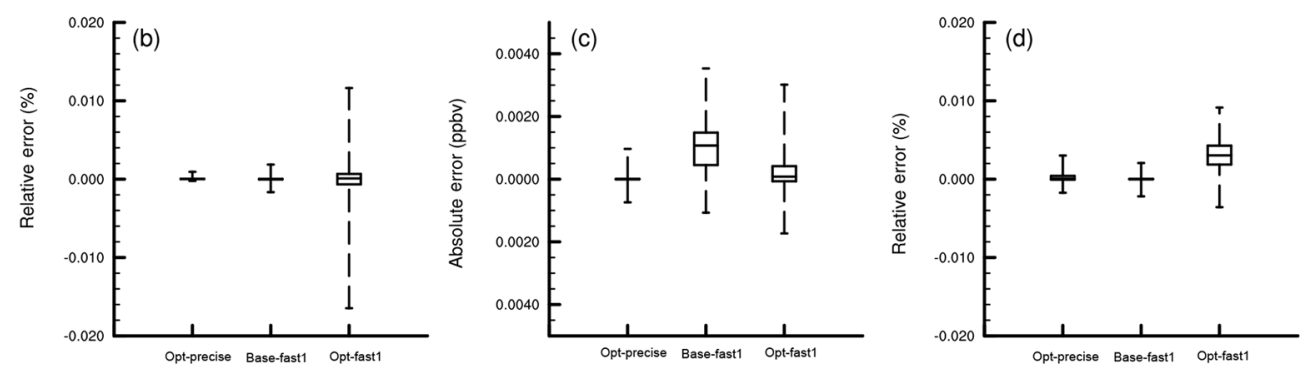

$\mathrm{SO}_{2}$
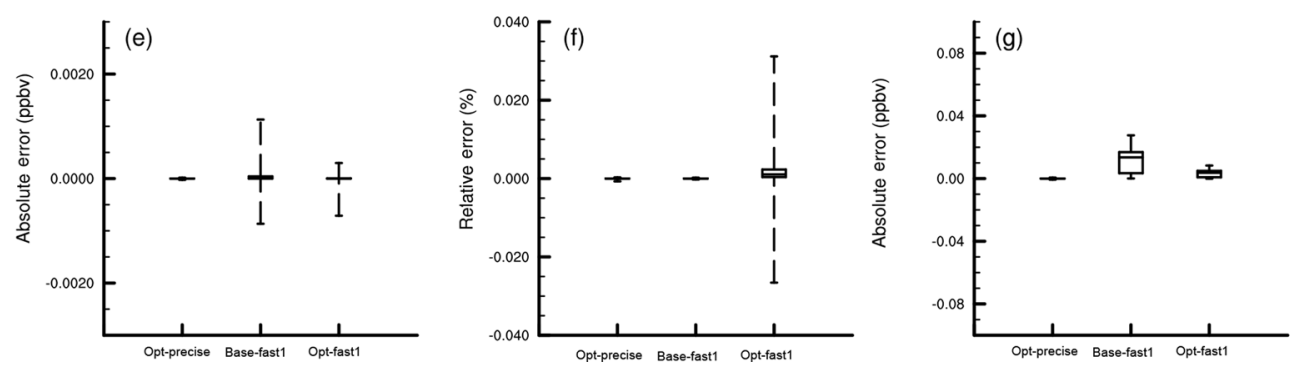

$\mathrm{CO}$

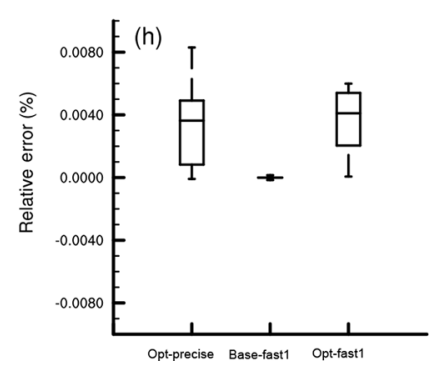

Figure 7. The distributions of absolute errors and relative errors for $\mathrm{O}_{3}, \mathrm{NO}_{2}, \mathrm{SO}_{2}$, and $\mathrm{CO}$ in the near-surface model layer. The reference points are $1 \%, 25 \%, 50 \%, 75 \%$, and $99 \%$.

module and whole NAQPMS. Such results illustrate that the optimization for vectorization improves the potential on existing hardware and the performance is highly improved even with the relatively strict precise compile flag, which prevents most vectorizations.

The new generation CPU further improves the performance of the MP CBM-Z. Using the platform with the new generation processor Xeon Gold 6132, the speedups reach $1.84 \times$ and $1.66 \times$ for the CBM-Z and the NAQPMS with the precise compile flag, respectively, and adopting the fast $=1$ compile flag improves the speedups to $8.22 \times$ and $3.50 \times$ compared with the benchmark performance. On the same Xeon Gold 6132 platform with the $f a s t=1$ compile flag, the MP CBM-Z gains 4.90 and 2.22 times acceleration compared with the baseline CBM-Z for the gas-phase chemistry module and the whole NAQPMS. Moreover, the proportion of time taken by the gas-phase chemistry declined to $30.74 \%$ compared to $72.26 \%$ in the baseline model. 
Table 6. The performance tests of the baseline and optimized code on the diverse platforms with different compile flags. The unit of the wall times for the tests is seconds (s).

\begin{tabular}{|c|c|c|c|c|c|c|c|}
\hline & $\begin{array}{l}\text { Vector } \\
\text { processor }\end{array}$ & Instruction & $\begin{array}{l}\text { Wall time } \\
\text {-fp-model }\end{array}$ & $\begin{array}{r}\text { Wall time } \\
(\mathrm{CBMZ})\end{array}$ & $\begin{array}{r}\text { Speedup } \\
\text { (Total) }\end{array}$ & $\begin{array}{l}\text { Speedup } \\
\text { (CBMZ) }\end{array}$ & (Total) \\
\hline \multirow{4}{*}{ Baseline NAQPMS } & \multirow{2}{*}{ Xeon E5-2680 V4 } & \multirow{2}{*}{ AVX2 } & precise & 17675.86 & 24460.54 & 1.00 & 1.00 \\
\hline & & & fast $=1$ & 13201.56 & 19619.20 & 1.34 & 1.25 \\
\hline & \multirow{2}{*}{ Xeon Gold 6132} & \multirow{2}{*}{ AVX512 } & precise & 13817.24 & 18950.95 & 1.28 & 1.29 \\
\hline & & & fast $=1$ & 10544.60 & 15502.39 & 1.68 & 1.58 \\
\hline \multirow{5}{*}{$\begin{array}{l}\text { NAQPMS with } \\
\text { MP CBM-Z }\end{array}$} & \multirow{2}{*}{ Xeon E5-2680 V4 } & \multirow{2}{*}{ AVX2 } & precise & 11127.90 & 17454.95 & 1.59 & 1.40 \\
\hline & & & fast $=1$ & 3971.48 & 10019.21 & 4.45 & 2.44 \\
\hline & \multirow{2}{*}{ Xeon Gold 6132} & \multirow{2}{*}{ AVX512 } & precise & 9584.59 & 14698.38 & 1.84 & 1.66 \\
\hline & & & fast $=1$ & 2150.20 & 6994.43 & 8.22 & 3.50 \\
\hline & Xeon Phi 7250 & AVX512 & fast $=1$ & 2997.96 & 19239.20 & 5.90 & 1.27 \\
\hline
\end{tabular}

In addition, the MP CBM-Z extends the benefit gained from advanced hardware. Using the same fast $=1$ compile option, the performance of the baseline CBM-Z on the AVX512 platform is about 1.25 times that on the AVX-2 platform, and the performance of the MP CBM-Z is about 1.84 times of that on AVX-2 platform. The efficiency of using the new CPUs improved by about $47 \%$ by adopting the MP CBM-Z. Therefore, enhancing the vectorization of code ensures that applications, like the CTM in this paper, could further utilize the improvement of processors on vectorization in the future.

KNL are more reliant on SIMD for performance according to the test results. The CBM-Z module is accelerated on KNL with a speedup of $5.9 \times$, but the whole model only achieved a 1.27 times acceleration compared with the benchmark performance. Comparing the baseline CBM-Z on the Intel Xeon Gold 6132 platform, the MP CBM-Z achieves a speedup of $3.52 \times$ for the gas-phase chemistry on KNL; however, the performance of the whole model declined by $24 \%$. Therefore, the MP CBM-Z largely improved the efficiency of CBM-Z on KNL by improving its vectorization, but further optimizations are required for greater efficiency of the whole CTM on the KNL architecture.

\section{Conclusions and discussion}

A new framework was designed for helping the chemical kinetics kernel CBM-Z to adapt to the next-generation processes by improving its vectorization. Through packing multiple spatial points, the optimized CBM-Z module handled these simultaneously. The functions in the original CBM-Z were restructured with loops, which provided the opportunity to implement the fine-grain level parallelization of vectorization. Meanwhile, we masked the heterogeneous grid boxes to integrate the chemistry sub-schemes in the CBM-Z to perform the calculation of multiple grid boxes simultaneously. Since the contiguous grid boxes have similar chemistry pro- cesses, the impact of this on the scientific performance was largely limited, and the code was highly vectorized.

The computation cluster equipped with two generation CPUs (Intel Xeon E5-2680 V4 and Intel Xeon Gold 6132) and KNL (Intel Xeon Phi 7250) provided by IAP, CAS, were used to test the performance. We tested the code with two different compile options of - $f p$-model precise and $-f p$ model fast $=1$ to present its impact on the accuracy of singleprecision computation and performance. The validation test ensured the reliability of our optimization on the model results, and the errors in all diagnostic chemical species caused by the single float calculations were lower than about $0.025 \%$ after $10 \mathrm{~h}$ integration with the fast $=1$ option. Based on the HPC performance characteristic from the Intel VTune tools on the Intel Xeon Gold 6132, the GFLOPS of CBM-Z increased from 4.81 to 21.37 , and the vector capacity usage improved from $14.30 \%$ in the baseline CBM-Z to $89.40 \%$ in the optimized CBM-Z.

The tests using the single core showed that the vectorization optimization led to speedups of $5.16 \times$ and $8.97 \times$ on Intel Xeon E5-2680 V4 and Intel Xeon Gold 6132 CPUs, respectively, and KNL achieves a speedup of $3.69 \times$ compared with the baseline CBM-Z on the Intel Xeon E5-2680 V4 platform. It highlights the importance of vectorization on the KNL platform. Meanwhile, we also tested the MPI and OpenMP version of CBM-Z. The speedup on the two generation CPUs can reach $104.63 \times$ and $198.50 \times$ using MPI and $101.02 \times$ and $194.60 \times$ using OpenMP, respectively. The speedup on the KNL node can reach $175.23 \times$ using MPI and $167.45 \times$ using OpenMP. The speedup of the optimized CBM-Z is approximately $40 \%$ higher on a one-socket KNL platform than on a two-socket Broadwell platform and about $13 \%-16 \%$ lower than on a two-socket Skylake platform.

The regional CTM NAQPMS was also used to test the practical improvement of the MP CBM-Z in more realistic scenarios. The baseline and optimized code of NAQPMS compiled with the precise and fast $=1$ options, respectively, 
were tested on diverse platforms. The model outputs after $72 \mathrm{~h}$ simulation were used to evaluate the error by the code as well as the compile flags. The difference between the baseline and optimized code are generally in the range of $\pm 0.02 \mathrm{ppbv}$ using precise. The maximum discrepancy over the whole map is about $0.166,0.197,0.001$, and $0.03 \mathrm{ppbv}$ for $\mathrm{NO}_{2}, \mathrm{O}_{3}, \mathrm{SO}_{2}$, and $\mathrm{CO}$. The fast=1 option leads to larger errors; however, computational performance could benefit a lot through adopting this option.

The results of the CTM test with the fast $=1$ option show that the MP CBM-Z leads to a speedup of 3.32 and 1.96 for the gas-phase chemistry module and the CTM on the Intel Xeon E5-2680 platform, respectively. Moreover, on the new Intel Xeon Gold 6132 platform, the MP CBM-Z gains $4.90 \times$ and $2.22 \times$ speedups for the gas-phase chemistry module and the whole CTM. For the KNL, the MP CBM-Z enables a $3.52 \times$ speedup for the gas-phase chemistry module, but the whole model lost $24.10 \%$ performance compared to the CPU platform due to the poor performance of other modules. Since this optimization seeks to improve the utilization of the VPU, the model is more suitable for the new generation processors adopting the more advanced SIMD technology. The results of our tests already show that the benefit of updating CPU improved by about $47 \%$ by using the MP CBM-Z since the optimized code has better adaptability for the new hardware.

In general, the choice of -fp-model compile flag decides the balance between accuracy and performance. According to our test, after using the fast=l option, the performance of the code is largely improved by sacrificing some accuracy. However, the loss of accuracy is relatively small, and in some practical applications that do not require high-accuracy floating-point calculations, it is acceptable to use the fast $=1$ option.

Besides the CBM- $Z$ chemical scheme, this algorithm is also suitable for models with a similar code structure to improve its vectorization. In addition, in this study, CBM-Z was treated as an example to describe this simple optimization strategy to implement the optimization on new generation processors, which emphasize the importance of vectorization. However, some specific strategies should also be considered before adoption. The optimizing methods such as constructing loops from the discrete scalar calculations as described in Wang et al. (2017), would diminish the readability of the source code by using indirect indexing and could cause problems to subsequent developers. Therefore, it is essential to adopt good practice, e.g., commenting code well and controlling the compile process, for ease of maintenance and development.

Code availability. The source code of the baseline and optimized version CBM-Z box model, including OpenMP and MPI versions, is available online via ZENODO (https://doi.org/10.5281/zenodo.1161576; Wang et al., 2018).
Supplement. The supplement related to this article is available online at: https://doi.org/10.5194/gmd-12-749-2019-supplement.

Author contributions. QW, HSC, XT, and ZFW planned and organized the project. JL and $\mathrm{HW}$ designed fine-grained parallelization algorithm for CBM-Z module, and QW HSC, XT, and XC took part in the discussion. JL, HW, HSC, and XT prepared the CBM$\mathrm{Z}$ test cases and input datasets, and HW, HSC, and QW finished box model validation. XT, HSC, and ZWF prepared the NAQPMS code and its input dataset, HW coupled the MP CBM-Z module to NAQPMS, and QW, HW, and XT validated and discussed the model results. HW and JL analyzed the model performance data, and QW, HQC, and LW validated its reasonability. HW and QW wrote the manuscript. HW, JL, QW, XT, HSC, and XC revised the manuscript. HSC, XT, ZFW, XC, HQC, and LW reviewed and provided key comments on the paper.

Competing interests. The authors declare that they have no conflict of interest.

Acknowledgements. The National Key R\&D Program of China (2017YFC0209805 and 2016YFB0200800), the CAS Information Technology Program (XXH13506-302), the National Natural Science Foundation of China (41305121), and the Fundamental Research Funds for the Central Universities funded this work. The authors would like to thank the Institute of Atmospheric Physics and Intel Corporation's Software Support Group (SSG) for providing the high-performance computing (HPC) environment and technical support. The authors thank the topic editor and three anonymous referees for their valuable comments.

Edited by: Fiona O'Connor

Reviewed by: three anonymous referees

\section{References}

Chang, J. S., Brost, R. A., Isaksen, I. S. A., Madronich, S., Middleton, P., Stockwell, W. R., and Walcek, C. J.: A three-dimensional Eulerian acid deposition model: Physical concepts and formulation, J. Geophys. Res.-Atmos., 92, 14681-14700, 1987.

Chen, H., Wang, Z., Qizhong, W. U., Jianbin, W. U., Yan, P., Tang, X., and Wang, Z.: Application of Air Quality Multi-Model Forecast System in Guangzhou: Model Description and Evaluation of $\mathrm{PM}_{10}$ Forecast Performance, Clim. Environ. Res., 18, 427-435, 2013.

Chen, H. S., Wang, Z. F., Li, J., Tang, X., Ge, B. Z., Wu, X. L., Wild, O., and Carmichael, G. R.: GNAQPMS-Hg v1.0, a global nested atmospheric mercury transport model: model description, evaluation and application to trans-boundary transport of Chinese anthropogenic emissions, Geosci. Model Dev., 8, 2857-2876, https://doi.org/10.5194/gmd-8-2857-2015, 2015.

Feng, F., Wang, Z., Li, J., and Carmichael, G. R.: A nonnegativity preserved efficient algorithm for atmospheric chemical kinetic equations, Appl. Mathe. Comput., 271, 519-531, 2015. 
Gao, M., Carmichael, G. R., Wang, Y., Saide, P. E., Yu, M., Xin, J., Liu, Z., and Wang, Z.: Modeling study of the 2010 regional haze event in the North China Plain, Atmos. Chem. Phys., 16, 1673-1691, https://doi.org/10.5194/acp-16-1673-2016, 2016.

Grell, G. A., Peckham, S. E., Schmitz, R., McKeen, S. A., Frost, G., Skamarock, W. C., and Eder, B.: Fully coupled "online" chemistry within the WRF model, Atmos. Environ., 39, 6957-6975, https://doi.org/10.1016/j.atmosenv.2005.04.027, 2005.

Guenther, A., Karl, T., Harley, P., Wiedinmyer, C., Palmer, P. I., and Geron, C.: Estimates of global terrestrial isoprene emissions using MEGAN (Model of Emissions of Gases and Aerosols from Nature), Atmos. Chem. Phys., 6, 3181-3210, https://doi.org/10.5194/acp-6-3181-2006, 2006.

Guenther, A. B., Jiang, X., Heald, C. L., Sakulyanontvittaya, T., Duhl, T., Emmons, L. K., and Wang, X.: The Model of Emissions of Gases and Aerosols from Nature version 2.1 (MEGAN2.1): an extended and updated framework for modeling biogenic emissions, Geosci. Model Dev., 5, 1471-1492, https://doi.org/10.5194/gmd-5-1471-2012, 2012.

Gurjar, B. R., Ravindra, K., and Nagpure, A. S.: Air pollution trends over Indian megacities and their localto-global implications, Atmos. Environ., 142, 475-495, https://doi.org/10.1016/j.atmosenv.2016.06.030, 2016.

Lawrence, B. N., Rezny, M., Budich, R., Bauer, P., Behrens, J., Carter, M., Deconinck, W., Ford, R., Maynard, C., Mullerworth, S., Osuna, C., Porter, A., Serradell, K., Valcke, S., Wedi, N., and Wilson, S.: Crossing the chasm: how to develop weather and climate models for next generation computers?, Geosci. Model Dev., 11, 1799-1821, https://doi.org/10.5194/gmd-111799-2018, 2018.

Li, J., Wang, Z., Wang, X., Yamaji, K., Takigawa, M., Kanaya, Y., Pochanart, P., Liu, Y., Irie, H., Hu, B., Tanimoto, H., and Akimoto, H.: Impacts of aerosols on summertime tropospheric photolysis frequencies and photochemistry over Central Eastern China, Atmos. Environ., 45, 1817-1829, https://doi.org/10.1016/j.atmosenv.2011.01.016, 2011.

Li, J., Wang, Z., Zhuang, G., Luo, G., Sun, Y., and Wang, Q.: Mixing of Asian mineral dust with anthropogenic pollutants over East Asia: a model case study of a superduststorm in March 2010, Atmos. Chem. Phys., 12, 7591-7607, https://doi.org/10.5194/acp-12-7591-2012, 2012.

Li, J., Wang, Z., Huang, H., Hu, M., Meng, F., Sun, Y., Wang, X., Wang, Y., and Wang, Q.: Assessing the effects of transboundary aerosol transport between various city clusters on regional haze episodes in spring over East China, Tellus B, 65, 20052, https://doi.org/10.3402/tellusb.v65i0.20052, 2013.

Linford, J. C., Michalakes, J., Vachharajani, M., and Sandu, A.: Multi-core acceleration of chemical kinetics for simulation and prediction, in: Proceedings of the Conference on High Performance Computing Networking, Storage and Analysis/ACM, 1420 November 2009, Portland, Oregon, USA, 1-11, 2009.

Mielikainen, J., Huang, B., and Huang, A. H.-L.: Intel Xeon Phi accelerated Weather Research and Forecasting (WRF) Goddard microphysics scheme, Geosci. Model Dev. Discuss., 7, 8941-8973, https://doi.org/10.5194/gmdd-7-8941-2014, 2014.

San José, R., Pérez, J. L., Balzarini, A., Baró, R., Curci, G., Forkel, R., Galmarini, S., Grell, G., Hirtl, M., Honzak, L., Im, U., Jiménez-Guerrero, P., Langer, M., Pirovano, G., Tuccella, P., Werhahn, J., and Žabkar, R.: Sensitivity of feedback effects in
CBMZ/MOSAIC chemical mechanism, Atmos. Environ., 115, 646-656, https://doi.org/10.1016/j.atmosenv.2015.04.030, 2015.

Seinfeld, J. H. and Pandis, S. N.: Atmospheric Chemistry and Physics: From Air Pollution to Climate Change, 2nd Edition, John Wiley \& Sons, New York, USA, 2012.

Sindelarova, K., Granier, C., Bouarar, I., Guenther, A., Tilmes, S., Stavrakou, T., Müller, J.-F., Kuhn, U., Stefani, P., and Knorr, W.: Global data set of biogenic VOC emissions calculated by the MEGAN model over the last 30 years, Atmos. Chem. Phys., 14, 9317-9341, https://doi.org/10.5194/acp-14-9317-2014, 2014.

Skamarock, W. C., Klemp, J. B., Dudhia, J., Gill, D. O., Barker, D. M., Duda, M. G., Huang, X.-Y., Wang, W., and Powers, J. G.: A description of the advanced research WRF version 3, NCAR Technical Note NCAR/TN-475+STR, 2008.

Sodani, A., Gramunt, R., Corbal, J., Kim, H. S., Vinod, K., Chinthamani, S., Hutsell, S., Agarwal, R., and Liu, Y. C.: Knights Landing: Second-Generation Intel Xeon Phi Product, IEEE Micro, 36, 34-46, 2016.

Wang, H., Chen, H., Wu, Q., Lin, J., Chen, X., Xie, X., Wang, R., Tang, X., and Wang, Z.: GNAQPMS v1.1: accelerating the Global Nested Air Quality Prediction Modeling System (GNAQPMS) on Intel Xeon Phi processors, Geosci. Model Dev., 10, 2891-2904, https://doi.org/10.5194/gmd-102891-2017, 2017.

Wang, H., Lin, J., Wu, Q., Chen, H., Tang, X., Wang, Z., Chen, X., and Cheng, H.g: Design a new architecture of CBMZ gasphase chemical mechanism for the next generation processors, https://doi.org/10.5281/zenodo.1161576, 2018.

Wang, Y., Chen, H., Wu, Q., Chen, X., Wang, H., Gbaguidi, A., Wang, W., and Wang, Z.: Three-year, $5 \mathrm{~km}$ resolution China $\mathrm{PM}_{2.5}$ simulation: Model performance evaluation, Atmos. Res., 207, 1-13, https://doi.org/10.1016/j.atmosres.2018.02.016, 2018.

Wang, Z., Maeda, T., Hayashi, M., Hsiao, L. F., and Liu, K. Y.: A Nested Air Quality Prediction Modeling System for Urban and Regional Scales: Application for High-Ozone Episode in Taiwan, Water Air Soil Pollut., 130, 391-396, https://doi.org/10.1023/A:1013833217916, 2001.

Wu, Q., Wang, Z., Gbaguidi, A., Tang, X., and Zhou, W.: Numerical Study of The Effect of Traffic Restriction on Air Quality in Beijing, Sola, 6, 17-20, 2010.

Wu, Q. Z., Xu, W. S., Shi, A. J., Li, Y. T., Zhao, X. J., Wang, Z. F., Li, J. X., and Wang, L. N.: Air quality forecast of PM10 in Beijing with Community Multi-scale Air Quality Modeling (CMAQ) system: emission and improvement, Geosci. Model Dev., 7, 2243-2259, https://doi.org/10.5194/gmd-7-2243-2014, 2014.

Xu, S., Huang, X., Oey, L.-Y., Xu, F., Fu, H., Zhang, Y., and Yang, G.: POM.gpu-v1.0: a GPU-based Princeton Ocean Model, Geosci. Model Dev., 8, 2815-2827, https://doi.org/10.5194/gmd8-2815-2015, 2015.

Zaveri, R. A. and Peters, L. K.: A new lumped structure photochemical mechanism for long-scale applications, J. Geophys. Res.Atmos., 104, 30387-30415, 1999.

Zhang, Q., Jiang, X., Tong, D., Davis, S. J., Zhao, H., Geng, G., Feng, T., Zheng, B., Lu, Z., Streets, D. G., Ni, R., Brauer, M., van Donkelaar, A., Martin, R. V., Huo, H., Liu, Z., Pan, D., Kan, H., Yan, Y., Lin, J., He, K., and Guan, D.: Transboundary health impacts of transported global 
air pollution and international trade, Nature, 543, 705-709, https://doi.org/10.1038/nature21712, 2017.

ZiFa, W., FuYing, X., XiQuan, W., JunLing, A., and Jiang, Z.: Development and Application of Nested Air Quality Prediction Modeling System, Chin. J. Atmos. Sci., 30, 778-790, 2006.
Zimmermann, J. and Poppe, D.: A Supplement for the RADM2 Chemical Mechanism: The Photooxidation of Isoprene, Atmos. Environ., 30, 1255-1269, 1994. 